\title{
Apraxia for differentiating Alzheimer's disease from subcortical vascular dementia and mild cognitive impairment
}

This article was published in the following Dove Press journal:

Neuropsychiatric Disease and Treatment

8 July 2013

Number of times this article has been viewed

\author{
Serhat Ozkan' \\ Demet Ozbabalik Adapinar' \\ Nese Tuncer Elmaci² \\ Didem Arslantas ${ }^{3}$ \\ 'Department of Neurology, Eskișehir \\ Osmangazi University Medical Faculty, \\ Eskisehir, Turkey; ${ }^{2}$ Department of \\ Neurology, Marmara University \\ Medical Faculty, Istanbul, Turkey; \\ ${ }^{3}$ Department of Public Health, \\ Eskișehir Osmangazi University \\ Medical Faculty, Eskisehir, Turkey
}

\begin{abstract}
Although ideomotor limb apraxia is considered to be a typical sign of cortical pathologies such as Alzheimer's disease (AD), it has been also reported in subcortical neurodegenerative diseases and vascular lesions. We aimed to investigate the difference between $A D$, subcortical vascular dementia ( $\mathrm{SVaD}$ ) and mild cognitive impairment $(\mathrm{MCI})$ patients by means of ideomotor limb apraxia frequency and severity. Ninety-six AD, $72 \mathrm{SVaD}$, and $84 \mathrm{MCI}$ patients were assessed with the mini-mental status examination (MMSE), clinical dementia rating (CDR) and the apraxia screening test of TULIA (AST). Apraxia was significantly more frequent in the $\mathrm{AD}$ patients (32.3\%) than in both of the $\mathrm{SVaD}(16.7 \%)$ and $\mathrm{MCI}(4.8 \%)$ patients. The frequency of apraxia was also significantly higher in $\mathrm{SVaD}$ patients than in MCI patients. AD patients had significantly lower apraxia scores than both $\mathrm{SVaD}$ and MCI patients. In addition, a significant difference was found between $\mathrm{SVaD}$ and MCI patients in terms of apraxia scores. These results suggest that the widespread belief of the association between apraxia and cortical dementias is not exactly correct. The significant difference between both of the dementia groups and the MCI patients suggests that the absence of apraxia can be an indicator for MCI diagnosis.
\end{abstract}

Keywords: apraxia, Alzheimer's disease, subcortical vascular dementia, mild cognitive impairment

\section{Introduction}

The two most common forms of senile dementia, Alzheimer's disease (AD) and vascular dementia (VaD), share many of the same clinical signs and symptoms. Both diseases are characterized by cognitive decline, functional deterioration and neuropsychiatric symptoms that may present as behavioral alterations. ${ }^{1}$ Vascular disease risk factors such as hypertension, diabetes mellitus, obesity, hypercholesterolemia and vascular changes in the white matter of the brain may not only be present in vascular dementia, but also in Alzheimer's disease. ${ }^{2,3}$ These shared features always cause difficulties for the differential diagnosis of these two diseases, especially for the insidious onset of subcortical vascular dementia (SVaD) due to small-vessel disease, which leads to lacunar infarcts and the demyelination of white matter and deep subcortical gray and white matter. Although it is generally accepted that episodic memory impairment, dyscalculia, agnosia, apraxia, and aphasia are more prominent in $\mathrm{AD}$ and that executive/attentional processing, semantic memory and visuospatial/perceptual function are more impaired in vascular dementia, especially in patients with $\mathrm{SVaD}$, there is a lack of a uniformly identified cognitive, clinical, imaging and pathological profile typical of SVaD. ${ }^{4,5}$

Ideomotor limb apraxia, which is the most common subtype of apraxia, is the disturbance of planning and the execution of motor activity without any dysfunction 
of the motor or sensory nervous system or the lack of understanding and motivation. ${ }^{6}$ Dysfunction has been conventionally localized on the left fronto-parieto-temporal cortex, which has been implicated in processing movements of relatively high complexity, planning and sequencing movements. Bihemispheric lesions of the deep white matter, the commissural tracts or the basal ganglia have also been reported as a cause of ideomotor apraxia..$^{7-10}$ These reports suggest that an interruption in the association or commissural tracts of relative cortical areas can also cause the symptom of ideomotor apraxia. It is still accepted as a diagnostically indicative sign for Alzheimer's disease. ${ }^{11}$

A small study reported that the frequency of apraxia in mild cognitive impairment patients was not different from that of the age-matched healthy population. Retrospective analyses have shown that only MCI patients who subsequently proceeded to a clinical diagnosis of AD were significantly slower than controls in completing sequential movement tasks despite unimpaired performance. ${ }^{12}$ These results suggested that the absence of apraxia can be one of the clinical indicators for differentiating MCI from the other types of dementia.

In this study, we hypothesized that presence of apraxia may be an indicator to differentiate $\mathrm{SVaD}$ and MCI patients from mild to moderate $\mathrm{AD}$ patients and we investigated the difference in ideomotor apraxia frequency amongst AD, $\mathrm{SVaD}$ and MCI patients and the relationship between the dementia severity and the presence of ideomotor apraxia in each group of cognitive disease patients.

\section{Materials and methods}

The patients' data were retrospectively gathered from two neurodegenerative diseases units (Eskişehir Osmangazi University Medical Faculty and Marmara University Medical Faculty Neurology Clinics), which were participating in the Turkuaz Alzheimer Working (TAÇ) Group. This Turkish web-based dementia registry includes neuropsyhiatric and cognitive scales that are commonly used for dementia assessment and is currently being used in 18 dementia referral centers across Turkey. ${ }^{13}$ In this registry, patients are recorded with their detailed neuropsychiatric assessments including the Neuropsychiatric Inventory (NPI). Ninety-six consecutive AD, 72 subcortical SVaD and $84 \mathrm{MCI}$ patients were recruited from the web-based data of the two centers. The study was approved by the local ethics committee. An informed consent had been taken from all patients for this registry.

The AD diagnosis was based on the DSM IV-TR and NINDS-ADRDA criteria. ${ }^{11} \mathrm{SVaD}$ patients met the criteria for vascular dementia also proposed by the DSM IV-TR and NINDS-AIREN. All of the patients showed at least two of the following focal neurological symptoms or signs: facial palsy, dysarthria, dysphagia, pathologic laughing or crying, weakness or sensory loss of limbs, hyperactive deep tendon reflexes, extensor plantar responses, rigidity of limbs, axial rigidity, bradykinesia, hemiplegic gait, stooped posture, shortstep gait, festinating gait, shuffling gait, decreased arm swing while walking, and multi-step turning. All of the patients also had "severe" ischemic changes associated with small-vessel disease. These changes were defined by high signal intensity (HSI) on T2-weighted or FLAIR images corresponding to grade 3 of the Fazekas criteria. ${ }^{14}$ Brain MRI scans confirmed the absence of structural lesions such as intracranial hemorrhage, tumor, traumatic brain injury, or hydrocephalus. Patients with any cortical or subcortical territory infarction were also excluded. Both amnestic and non-amnestic MCI patients were included. The diagnosis of MCI was consistent with the criteria proposed by Petersen and colleagues. ${ }^{15}$ All of the AD patients were in the mild to moderate stage of the disease according to the Global Deterioration Scale/ Functional Assessment Staging system. ${ }^{16}$

Apraxia was examined using the Apraxia Screening Test of TULIA (AST), which has high diagnostic accuracy (95\% sensitivity and $100 \%$ specificity) in stroke. ${ }^{17}$ The AST requires the performance of 12 gestures in two domains: (1) imitation including one meaningless gesture, one intransitive (communicative) gesture and five transitive (tool-related) gestures and (2) pantomime including two intransitive gestures and three transitive gestures. The AST is a bedside test, and its administration requires approximately 3 minutes. Both arms were tested separately. The performance was dichotomously (fail: 0 , pass: 1 ) scored by the investigators immediately after the patient's performance. The scoring method is described in detail elsewhere. ${ }^{17} \mathrm{~A}$ validated Turkish version of the mini-mental status examination (MMSE) was used to evaluate the cognitive status. ${ }^{18}$ The clinical dementia rating scale was applied to all of the patients for staging the severity of the dementia. ${ }^{19}$

SPSS 15 (SPSS Inc, Chicago, IL, USA) was used for statistical analyses. Continuous variables were expressed as mean $\pm \mathrm{SD}$ and were compared between the patients with and without prior stress tests using the one-way ANOVA test with the post-hoc Tukey test. Categorical variables were expressed as frequency percentages and were compared between the groups using the chi-square test or the Fisher exact test as appropriate. ${ }^{20}$ Statistically significant $\alpha$ level was accepted as 0.05 and it was two-sided. 


\section{Results}

No significant differences were found between the AD and SVaD groups for each demographic group. There were no significant differences amongst the three groups in terms of sex ratio and age. Compared to the $\mathrm{AD}$ and $\mathrm{SVaD}$ groups, the MCI group had significantly higher MMSE scores and lower disease duration and $\mathrm{CDR}(P<0.001$, for each variable) (Table 1). Although MMSE and CDR scores were better in the $\mathrm{SVaD}$ group than in the $\mathrm{AD}$ group, the difference was found to be insignificant $(P>0.05$, for each variable).

Apraxia was significantly more frequent in $\mathrm{AD}$ disease patients $(32.3 \%, \mathrm{n}=31)$ than both $\operatorname{SVaD}(16.7 \%, \mathrm{n}=12)$ $\left(X^{2}=4.787\right.$ at $\left.\mathrm{df}=1, P=0.0287\right)$ and MCI $(4.8 \%, \mathrm{n}=4)$ $\left(X^{2}=18.778\right.$ at $\left.\mathrm{df}=1, P<0.005\right)$ patients. The frequency of apraxia was also significantly higher in $\mathrm{SVaD}$ patients than the MCI patients $\left(X^{2}=6.545\right.$ at $\left.\mathrm{df}=1, P=0.0105\right)$. When we compared the groups according to the apraxia scores, we observed that $\mathrm{AD}$ patients had significantly lower apraxia scores than both of the SVaD and MCI patients (odds ratio: 5.04 [95\% Confidence interval $\{\mathrm{CI}\}: 1.84-11.73], P<0.05$ and odds ratio: 4.17 [95\% CI: 2.67-9.87], $P<0.001$, respectively). In addition, a significant difference was found between the SVaD and MCI patients in terms of apraxia scores (odds ratio: 3.12 [\%95 CI: 1.12-5.71], $P<0.05$ ) (Figure 2).

\section{Discussion}

Although a large number of studies have revealed many aspects of the pathophysiology of $\mathrm{AD}$, no strongly specific biological or laboratory markers for this disease have been detected. This paucity is a possible reason for low diagnostic accuracy, especially in the early stages of the disease in which the disease is often confused with other forms of dementia, particularly vascular dementia. ${ }^{21,22}$ With the lack of a specific diagnostic marker, the diagnosis is still based primarily on clinical findings. The most common and prominent presentation is the impairment of anterograde episodic

Table I Demographic data of the study population

\begin{tabular}{llll}
\hline & AD patients & SVaD & MCI \\
\hline Number of patients & 96 & 72 & 84 \\
Sex (male/female) & $4 \mathrm{I} / 55$ & $32 / 40$ & $43 / 4 \mathrm{I}$ \\
Age (years \pm SD) & $73.32 \pm \mathrm{II} .43$ & $74.43 \pm \mathrm{II} .84$ & $68.66 \pm 9.97$ \\
Disease duration & $6.1 \pm 3.2$ & $5.4 \pm 4.1$ & $2.1 \pm 1.9^{\mathrm{a}}$ \\
(years \pm SD) & & & \\
CDR & $1.19 \pm 0.84$ & $0.99 \pm 0.7 \mathrm{I}$ & $0.44 \pm 0.19^{\mathrm{b}}$ \\
MMSE & $17.16 \pm 8.45$ & $19.4 \pm 7.72$ & $25.42 \pm 4.1 \mathrm{I}^{\mathrm{b}}$ \\
\hline
\end{tabular}

Notes: $\mathrm{a} P<0.05$; $\mathrm{b} P<0.001$.

Abbreviations: $A D$, Alzheimer's disease; $S V a D$, subcortical vascular dementia; $\mathrm{MCl}$, mild cognitive impairment; SD, standard deviation; CDR, clinical dementia rating; MMSE, mini-mental status examination.

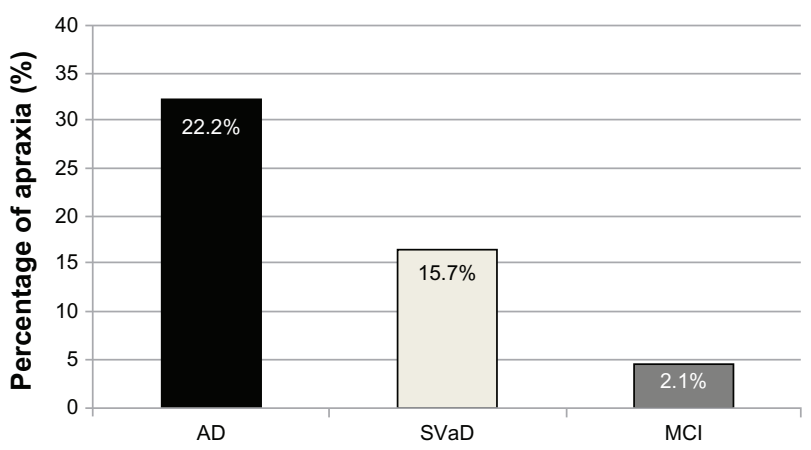

Figure I Apraxia frequency in $\mathrm{AD}, \mathrm{SVaD}$ and $\mathrm{MCl}$ patients. Abbreviations: $\mathrm{AD}$, Alzheimer's disease; $\mathrm{SVaD}$, subcortical vascular dementia; $\mathrm{MCl}$, mild cognitive impairment.

memory. The disease progression over time leads to involvement of other cognitive domains in addition to memory, including visuospatial function and praxis, language, and executive skills. ${ }^{23}$ In our study with $\mathrm{AD}$ and SVaD patients with similar disease duration and dementia severity scores, apraxia seems to be a significant differential marker between these diseases, but it must be noted that $\mathrm{SVaD}$ patients can also have significant ideomotor apraxia. Therefore, apraxia, unaccompanied by other signs of dementia, is a weak differential diagnosis parameter for $\mathrm{AD}$ and $\mathrm{SVaD}$.

Although apraxia is accepted as one of the diagnostic clinical variables for $\mathrm{AD}$, there are a few studies about its diagnostic value. ${ }^{24-28}$ In one of the early studies, Kramer et al ${ }^{25}$ investigated the frequency of apraxia with agnosia and aphasia in groups of cortical (probable AD) dementias and a heterogeneous group of subcortical dementias (Parkinson's disease and normal pressure hydrocephalus); they reported that only aphasia had been significantly more frequent in cortical dementias and had a very low diagnostic sensitivity, specificity and total predictive value for all three symptoms. These authors concluded that cortical and subcortical dementias cannot be reliably dissociated on the basis of apraxia, aphasia or agnosia. In a similar recent report assessing the occurrence of ideomotor

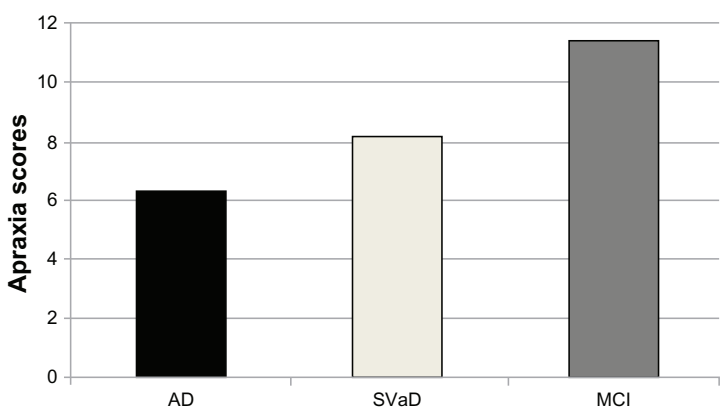

Figure 2 Mean apraxia scores of $\mathrm{AD}, \mathrm{SVaD}$ and $\mathrm{MCl}$ patients. Abbreviations: $A D$, Alzheimer's disease; $S V a D$, subcortical vascular dementia; $\mathrm{MCl}$, mild cognitive impairment. 
apraxia in AD and Huntington disease patients, the authors concluded that ideomotor apraxia was a common sign in both groups of patients. Furthermore, they reported that significantly more Huntington disease patients had been classified as apraxic - according to the hands' and finger' imitation and pantomimic subtypes - than AD patients. ${ }^{26}$ However, our study found that apraxia was more frequent in $\mathrm{AD}$ than the SVaD patients. This inconsistency between our results and those of the other subcortical dementia studies could be the result of the difference in the pathogenesis and lesion localization of neurodegenerative and vascular subcortical dementias. For example, in Huntington disease, prominent heterogeneously cortical dysfunction overlapping with the brain areas of praxis function, even in early stages of the disease, can be a different pathological process from SVaD. ${ }^{29-31}$ As the authors of the Huntington disease study concluded, the cortical degeneration during the disease process may contribute highly to the development of apraxia. ${ }^{26}$ Previous studies have reported that thalamic and basal ganglia vascular lesions were more associated with apraxia. ${ }^{32}$ However, our SVaD group had heterogeneous subcortical lesion localization, and the basal ganglia lesions were not separately investigated in the present study. This approach could be the reason for the difference between our results and those of previous neurodegenerative dementia studies. Significantly lower apraxia scores in AD patients than in SVaD patients with similar disease duration may suggest that praxis function is affected earlier and more severely in the neurodegenerative process. Without any pathologically proven diagnosis, however, our study is insufficient to make this conclusion.

In our study, the frequency of apraxia in SVaD patients (16.7\%) was significantly higher than in MCI (4.8\%) patients. Although there is insufficient data in the literature, the frequency of apraxia in subcortical stroke patients was reported to be $33.3 \%$ by a previous study. ${ }^{33}$ The authors of that study used a different apraxia test, the Movement Imitation Test, to assess the patients, and they included all of the stroke patients regardless of the presence of dementia. ${ }^{34}$ This methodological difference could explain the frequency disparity between our results and those of that study. These findings may suggest a methodological problem in the assessment methods for apraxia or the difference between the assessed groups of patients between the studies. For example, apraxia is a wellrecognized diagnostic sign in $\mathrm{AD}$, but the exact frequency is still controversial. The frequency has a wide range, from $33 \%$ to $77 \%$. $^{35,36}$ The assessment tests used in the studies, the stage of illness, disease severity, and the psychological status of patients can influence the results.
The apraxia assessment method may be the limitation of this study. We used a screening test called the Apraxia Screening of TULIA (AST) that comprises 12 items extracted from the more comprehensive test of upper limb apraxia, TULIA by item reduction analysis. ${ }^{17}$ The AST is a simple bedside test that was previously validated for stroke and Parkinson's disease patients with high clinimetric standards. ${ }^{17,37}$ The validation for both vascular and neurodegenerative disease patients was the reason for selecting this test for our study. Although a high diagnostic sensitivity and specificity for the presence of apraxia in stroke and Parkinson's disease patients have been reported, the reliability of this test in dementia may be questionable. Reliable results of screening for apraxia in multiple sclerosis have been reported in a recent study. ${ }^{38}$ However, it must be kept in mind that this test is only a fast screening test and does not allow for the subclassification of limb apraxia.

This study was designed as a retrospective, recorded data analyzing study. This can also be a limitation for our results, but it should be noted that all the data have been recorded by specialists in cognitive disorders. Besides the study gives data only from the Turkish population and the results may need to be re-evaluated in different cultures. Absence of a control group that could give information about the apraxia in a normal population, may be another limitation for the study. But we believe that the significant difference for MCI patients seems to be an apperant indicator.

\section{Conclusion}

In this study, we investigated the frequency and severity of ideomotor apraxia amongst three different cognitive problems (AD, SVaD and MCI) in addition to whether it is a good clinical marker to differentiate these diseases. Although ideomotor apraxia was observed more commonly and was more severe in the $\mathrm{AD}$ patients than in the $\mathrm{SVaD}$ and $\mathrm{MCI}$ patients, this significant difference was also observed between the SVaD and MCI patients. These results suggest that, contrary to widespread belief, it is not completely accurate to state that apraxia and cortical dementias are associated but do not include subcortical dementias. However, the significant difference between both of the dementia groups and MCI patients suggests that the absence of apraxia can only be a predictor for the diagnosis of MCI and that apraxia can be an additional supporting sign for the existing diagnostic criteria.

\section{Disclosure}

Authors have no conflicts of interest to report. 


\section{References}

1. Small G. Meeting the challenges of vascular dementia. Introduction. Int J Clin Pract. Suppl 2001;120:1-4.

2. Grammas P. Neurovascular dysfunction, inflammation and endothelial activation: implications for the pathogenesis of Alzheimer's disease. J Neuroinflammation. 2011;25:8-26.

3. Hachinski V. Preventable senility: a call for action against the vascular dementia. Lancet. 1992;340:645-648.

4. Looi JC, Sachdev PS. Differentiation of vascular dementia from AD on neuropsychological tests. Neurology. 1999;53:670-678.

5. Chan M, Lim WS, Sahadevan S. Stage-independent and stage-specific phenotypic differences between vascular dementia and Alzheimer's Disease. Dement Geriatr Cogn Disord. 2008;26:513-521.

6. Heilman KM, Rothi LJG. Apraxia. In: Heilman KM, Valenstein E, editors. Clinical Neuropsychology. New York: Oxford University Press, 2003:215-235.

7. Basso A, Luzzatti C, Spinnler H. Is ideomotor apraxia the outcome of damage to welldefined regions of the left hemisphere? Neuropsychological study of CAT correlation. J Neurol Neurosurg Psychiatry. 1980;43:118-126.

8. Jacobs DH, Adair JC, Macauley B, et al. Apraxia in corticobasal degeneration. Brain Cogn. 1999;40:336-354.

9. Hamilton JM, Haaland KY, Adair JC, Brandt J. Ideomotor limb apraxia in Huntington's disease: implications for corticostriate involvement. Neuropsychologia. 2003;41:614-621.

10. Agostoni E, Coletti A, Orlando G, Tredici G. Apraxia in deep cerebral lesions. J Neurol Neurosurg Psychiatry. 1983;46:804-808.

11. American Psychiatric Association, Diagnostic and Statistical Manual of Mental Disorders: DSM-IV-TR. 4th ed. Text Revision ed, Washington, DC, American Psychiatric Association, 2000.

12. Crutch SJ, Rossor MN, Warrington EK. A novel technique for the quantitative assessment of apraxic deficits: application to individuals with mild cognitive impairment. J Neuropsychol. 2007;1:237-257.

13. Yener GG; Turkuaz Alzheimer Working (TAC) Group. The neuropsychiatric inventory scores change across the mini mental state examination ranges in patients with Alzheimer's disease: a multicenter study in Turkey. Cogn Behav Neurol. 2009;22:264-269.

14. Fazekas F, Chawluk JB, Alavi A, Hurtig HI, Zimmerman RA. MR signal abnormalities at $1.5 \mathrm{~T}$ in Alzheimer's dementia and normal aging. Am J Roentgenol. 1987;149:351-356.

15. Petersen RC, Doody R, Kurz A, et al. Current concepts in mild cognitive impairment. Arch Neurol. 2001;58:1985-1992.

16. Auer S, Reisberg B. The GDS/FAST staging system. Int Psychogeriatr. 1997;9 Suppl 1:167-171.

17. Vanbellingen T, Kersten B, Van de Winckel A, et al. A new bedside test of gestures in stroke: the apraxia screen of TULIA (AST). Neurol Neurosurg Psychiatry. 2011;82:389-392.

18. Keskinoglu P, Ucku R, Yener G, Yaka E, Kurt P, Tunca Z. Reliability and validity of revised Turkish version of Mini Mental State Examination (rMMSE-T) in community-dwelling educated and uneducated elderly. Int J Geriatr Psychiatry. 2009;24:1242-1250.

19. Morris JC. The Clinical Dementia Rating (CDR): current version and scoring rules. Neurology. 1993;43:2412-2414.
20. Hinkle D,Wiersma W, Jurs S. Applied Statistics for the Behavioral Sciences. Chicago, Rand McNally, 1979.

21. Geldmacher DS. Differential diagnosis of dementia syndromes. Clin Geriatr Med. 2004;20:27-43.

22. White L, Small BJ, Petrovitch H, et al. Recent clinical-pathologic research on the causes of dementia in late life: update from the HonoluluAsia Aging Study. J Geriatr Psychiatry Neurol. 2005;18:224-227.

23. Albert MS. Changes in cognition. Neurobiol Aging. 2011; Suppl 1:S58-S63.

24. Koedam EL, Lauffer V, van der Vlies AE, van der Flier WM, Scheltens P, Pijnenburg YA. Early-versus late-onset Alzheimer's disease: more than age alone. J Alzheimer's Dis. 2010;19:1401-1408.

25. Kramer JH, Duffy JM. Aphasia, apraxia, and agnosia in the diagnosis of dementia. Dementia. 1996;7:23-26.

26. Holl AK, Ille R, Wilkinson L, et al. Impaired ideomotor limb apraxia in cortical and subcortical dementia: a comparison of Alzheimer's and Huntington's disease. Neurodegener Dis. 2011;8:208-215.

27. Capone JG, Della Sala S, Spinnler H, Venneri A. Upper and lower face and ideomotor apraxia in patients with Alzheimer's disease. Behav Neurol. 2003;14:1-8.

28. Siri S, Benaglio I, Frigerio A, Binetti G, Cappa SF. A brief neuropsychological assessment for the differential diagnosis between frontotemporal dementia and Alzheimer's disease. Eur J Neurol. 2001;8:125-132.

29. Rosas HD, Salat DH, Lee SY, et al. Cerebral cortex and the clinical expression of Huntington's disease: complexity and heterogeneity. Brain. 2008;131:1057-1068.

30. Nopoulos PC, Aylward EH, Ross CA, et al. Cerebral cortex structure in prodromal Huntington disease. Neurobiol Dis. 2010;40:544-554.

31. Thu DC, Oorschot DE, Tippett LJ, et al. Cell loss in the motor and cingulate cortex correlates with symptomatology in Huntington's disease. Brain. 2010;133:1094-1110.

32. De Renzi E, Faglioni P, Scarpa M, Crisi G. Limb apraxia in patients with damage confined to the left basal ganglia and thalamus. J Neurol Neurosurg Psychiatry. 1986;49:1030-1038.

33. Tabaki NE, Vikelis M, Besmertis L, Vemmos K, Stathis P, Mitsikostas DD. Apraxia related with subcortical lesions due to cerebrovascular disease. Acta Neurol Scand. 2010;122:9-14.

34. De Renzi E, Motti F, Nichelli P. Imitating gestures: a quantitative approach to ideomotor apraxia. Arch Neurol. 1980;37:6-10.

35. Della Sala S, Lucchelli F, Spinnler H. Ideomotor apraxia in patients with dementia of Alzheimer type. J Neurol. 1987;234:91-93.

36. Derouesné C, Lagha-Pierucci S, Thibault S, Baudouin-Madec V, Lacomblez L. Apraxic disturbances in patients with mild to moderate Alzheimer's disease. Neuropsychologia. 2000;38:1760-1769.

37. Vanbellingen T, Lungu C, Lopez G, et al. Short and valid assessment of apraxia in Parkinson's disease. Parkinsonism Relat Disord. 2012;18:348-350.

38. Kamm CP, Heldner MR, Vanbellingen T, Mattle HP, Müri R, Bohlhalter S. Limb apraxia in multiple sclerosis: prevalence and impact on manual dexterity and activities of daily living. Arch Phys Med Rehabil. 2012;93:1081-1085.
Neuropsychiatric Disease and Treatment

\section{Publish your work in this journal}

Neuropsychiatric Disease and Treatment is an international, peerreviewed journal of clinical therapeutics and pharmacology focusing on concise rapid reporting of clinical or pre-clinical studies on a range of neuropsychiatric and neurological disorders. This journal is indexed on PubMed Central, the 'PsycINFO' database and CAS.

\section{Dovepress}

The manuscript management system is completely online and includes a very quick and fair peer-review system, which is all easy to use. Visit http://www.dovepress.com/testimonials.php to read real quotes from published authors. 